Apidologie, 1987, 18 (2), 137-146

\title{
ÜBERPRÜFUNG DER SCHÄTZMETHODE ZUR ERMITTLUNG DER BRUTFLÄCHE UND DER ANZAHL ARBEITERINNEN IN FREIFLIEGENDEN BIENENVÖLKERN
}

\author{
Anton IMDORF, Georges BUEHLMANN, Luzio GERIG, Verena KILCHENMANN \\ und Hans WILLE \\ Forschungsanstalt für Milchwirtschaft \\ Sektion Bienen, CH-3097 Liebefeld-Bern, Schweiz
}

ZUSAMMENFASSUNG

Die « Liebefelder-Methode » zur Erfassung des Anzahl Arbeiterinnen, der offenen und gedeckelten Brutfläche wurde 1984 erneut geprüft. Die Erhebungen an zwei freifliegenden Völkern wurden vom April bis Oktober alle 3 Wochen durchgeführt. Zahl der Bienen und Brutfläche wurden geschätzt und anschliessend durch Wägen resp. Ausplanimetrieren nachkontrolliert. Die Schätz- und Messergebnisse korrelieren sehr gut für die Anzahl Bienen $\left(r^{2}=0.967 ; n=18\right)$ und für die gedeckelte Brut $\left(r^{2}=0.987\right)$. Die Schätzung der offenen Brutfläche korreliert weniger gut mit den Messungen $\left(r^{2}=0.654\right)$. Das Total der pro Volk während der ganzen Versuchsperiode geschätzten Brutfläche war um $1.6 \%$ resp. $5.4 \%$ zu hoch. Die Anzahl Bienen wurde regelmässig unterschätzt, was korrigiert werden kann, wenn die Standardbesetzung einer Wabenseite (Schweizermass, $921 \mathrm{~cm}^{2}$ ) mit 1200 anstatt 1100 Bienen berechnet wird. Diese Methode erlaubt es, den Massenwechsel des Volkes genau, rasch und mit geringer Störung der Bienen zu erfassen.

\section{EINLEITUNG}

Es hat nicht an Bestrebungen gefehlt, die Brutaktivität und in wenigen Fällen auch die Fluktuationen der Arbeiterinnenpopulation in Bienenvölkern zu erfassen. B.v. BERLEPSCH hatte bereits am 28. Juni 1853 sämtliche belegten Arbeiterinnenzellen eines Bienenvolkes ausgezählt (Brünnich, 1922). In der gleichen Arbeit veröffentlichte BRUENNICH eigene Brutmessungen von mehreren Völkern über die ganze Bienensaison. VoN EBERT (1922) stellte mit Hilfe von Brut- und Waagmessungen Berechnungen zum Massenwechsel des Bienenvolkes an. Weitere Messungen über die Brutentwicklung machte NoLAN (1925), sowie später viele andere Autoren. 
Farrar (1937) untersuchte den Einfluss der Volksstärke auf die Honigernte mittels Bienenwägung. Diese Methode ist sehr genau, wenn man wie FARRAR, bei jeder Messung pro Volk das durchschnittliche Gewicht der Einzelbiene mitbestimmt. Sie ist aber mit einem grossen Arbeitsaufwand verbunden. JEFFREE (1951) entwickelte eine Schätzmethode, indem er jede mit Bienen besetzte Wabenseite mit Fotografien verglich, deren Bienenzahl bekannt war. Er verfolgte 1955 als erster mit dieser Methode und mit Wägungen während der ganzen Bienensaison in regelmässigen Abständen die Entwicklung der Arbeiterinnenpopulation verschiedener Völker. Brutflächen schätzte er 1958 mit Hilfe eines Rasters in der Grösse der verwendeten Brutrahmen.

Ausgehend von den Arbeiten von Jefrree entwickelten Wille und Gerig anfangs der siebziger Jahre die in der vorliegenden Arbeit überprüfte « Liebefelder-Schätzmethode " zur Erfassung der Anzahl Arbeiterinnen und der offenen und gedeckelten Brutflächen eines Volkes (WILle und Gerig, 1976 ; GerIG, 1983). Schätzungen in regelmässigen Abständen von 21 Tagen erlauben eine objektive Erfassung des Massenwechsels der Bienenvölker.

\section{MATERIAL UND METHODEN}

Die Schätzmethode überprüften wir an den Völkern 4 und 8 auf dem Stand des Institutes in Liebefeld. Die Völker waren in Schweizerkasten (Hinterlader, Warmbau) in einem Bienenhaus untergebracht. Ihre Volksstärke wurde vom 17. April bis 1. Oktober 1984 neunmal in Intervallen von drei Wochen geschätzt.

\subsection{Schätzmethode}

Die Schätzung wurde frühmorgens vor Flugbeginn, in einem Raum durchgeführt, aus dem keine Bienen abfliegen konnten.

Bienen

Wir gingen davon aus, dass auf einer dicht besetzten Brutwabe im Schweizermass (Innenmasse : Höhe $34.5 \mathrm{~cm}$, Breite $26.7 \mathrm{~cm}$, Fläche $921 \mathrm{~cm}^{2}$ ) pro Wabenseite inklusiv Rahmen rund 1100 Bienen sitzen, dies entspricht ungefähr 120 dichtsitzenden Bienen pro $\mathrm{dm}^{2}$. Sind die Waben nur teilweise bedeckt, so wird gedanklich die dicht bedeckte Fläche bestimmt, z.B. bei halber Bedeckung ergibt dies 550 Bienen, bei einem Viertel ca. 250-300 Bienen, etc. (kleinste geschätzte Einheit : 50 Bienen). Das Ergebnis wird für jede Wabenseite notiert.

Mehr Schwierigkeiten bietet das Schätzen der Bienen, die sich manchmal knäuelartig am unteren Wabenschenkel oder an Kastenwänden und Kastenböden (vor allem in Hochböden) aufhalten. Bei einfacher dichter Bedeckung in der Grösse einer Brutwabe werden ebenfalls 1100 Bienen geschätzt. Bei mehrfacher Bedeckung sind entsprechende Zuschläge zu machen. Grössere Knäuel von Bienen, wie z.B. Bienenbärte am Kasteneingang oder im Kastenhochboden sollten zur Uebung über das Gewicht erfasst werden.

Brut

Geschätzt werden die offenen und gedeckelten Brutflächen der Arbeiterinnenbrut pro Wabenseite in $\mathrm{dm}^{2}$ (kleinste geschätzte Einheit : $0.1 \mathrm{dm}^{2}$ ). Daraus wird anschliessend die Anzahl der besetzten 
Brutzellen berechnet (400 Brutzellen pro $\mathrm{dm}^{2}$ ). Dieser Wert kann je nach Mittelwandprägung etwas höher oder etwas tiefer liegen. Dic geschätzte Brutfläche sollte periodisch mit einem Rasterrahmen (10 $\mathrm{cm} \times 10 \mathrm{~cm}$ Einteilung) oder mit einem Rollmeter überprüft werden.

\subsection{Wägung und Messung als Kontrolle}

Die Messungen wurden jeweils sofort nach der Schätzung durchgeführt.

Bienen

Sämtliche Bienen von den Waben und aus der Beute, sowie die abgeflogenen an den Fenstern wurden sorgfältig in eine tarierte Schwarmkiste abgewischt und gewogen. Daraus wurde eine Mischprobe von ca. 250 Bienen entnommen und das durchschnittliche Gewicht einer Biene ermittelt. Aus dem Gesamtgewicht wurde anschliessend die Zahl der Bienen des betreffenden Volkes berechnet. Die Drohnenbrut wurde in beiden Versuchsvölkern jeweils vor dem Schlüpfen ausgeschnitten.

Brut

Die Umrisse der offenen und gedeckelten Brutflächen wurden Wabenseite für Wabenseite auf Klarsichtfolien aufgezeichnet. Bei nicht geschlossenen Brutnestern wurde der Anteil der besetzten Zellen geschätzt. Mit einem Videoplan-Planimeters wurde die genaue Brutfläche ausgemessen und die Anzahl Brutzellen, unter Berücksichtigung des Anteils der besetzten Zellen, berechnet.

\section{ERGEBNISSE UND DISKUSSION}

\subsection{Anzahl Bienen}

Die Wägung ergab regelmässig eine höhere Anzahl Bienen als die Schätzung (Abb. 1 und 2). Die Völker 4 und 8 wurden im Durchschnitt der 9 Messungen um 15.5 resp. $12.1 \%$ unterschätzt. Diese Unterschätzung lässt sich wie folgt erklären :

1. Da durchwegs unterschätzt wurde, muss angenommen werden, dass der Standard von 1100 Bienen pro dicht besetzte Wabenseite zu tief angesetzt war. Seither haben wir als Standard 1200 Bienen pro Seite einer Schweizerwabe festgesetzt.

2. Das Verhalten der Bienen auf den Waben wurde beim Schätzen zu wenig berücksichtigt. Es zeigte sich, dass der Schätzfehler immer grösser war, wenn ein Teil der Bienen Kopf an Kopf Futter aus den Zellen aufnahm. Dieses Verhalten ist beim gleichen Volk von Messung zu Messung sehr unterschiedlich und muss daher vom Schätzer mitberücksichtigt werden. Bewegen sich die Bienen auf den Waben, so ist bei dichtem Besatz mit ca. 130 Bienen pro $\mathrm{dm}^{2} \mathrm{zu}$ rechnen. BurgetT und Burikam (1985) rechneten mit 138 Bienen pro $\mathrm{dm}^{2}$. Stecken die Bienen aber Kopf an Kopf in den Zellen, so kann sich diese Zahl bis auf 350 erhöhen. 


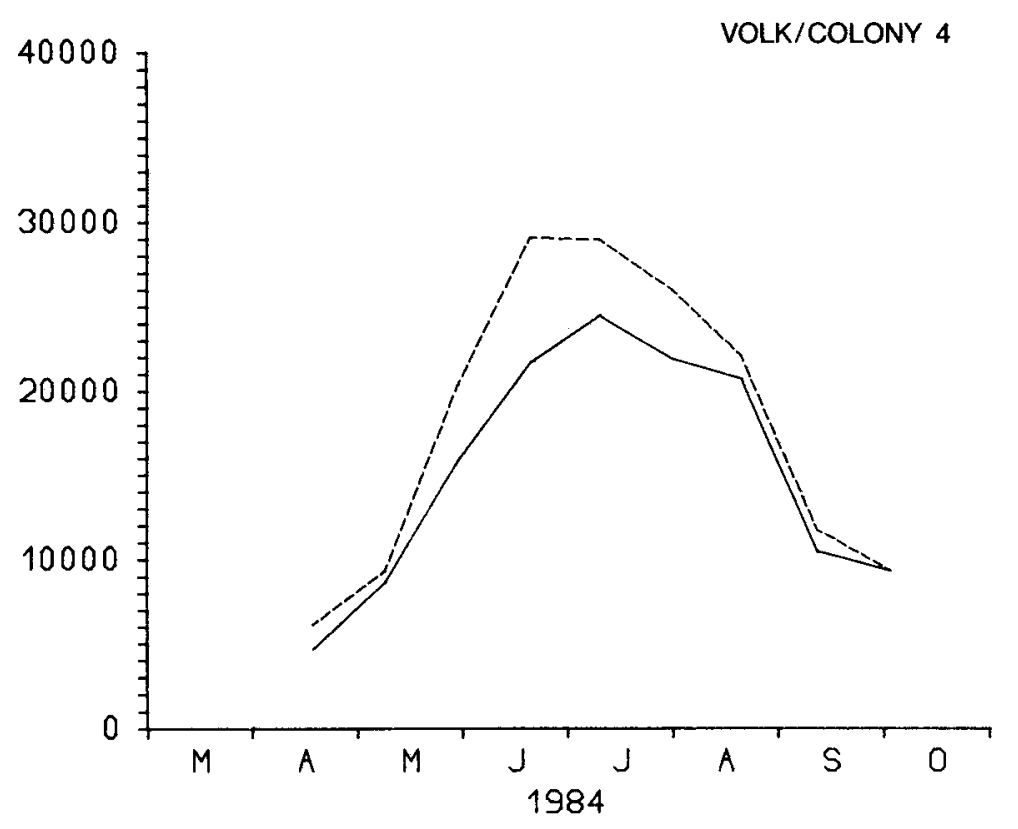

Aв8. 1. - Geschätzte (- - ) und durch Wägen bestimmt (-....) Anzahl Bienen

Fig. 1. - Number of bees obtained by estimation (- $\longrightarrow$ ) and weighing (-...--

Trotz dieser Unterschätzung ist die Genauigkeit der Schätzmethode zur Erfassung der Anzahl Bienen beachtlich. Werden die Schätzwerte den entsprechenden Messwerten gegenübergestellt, so ergibt sich eine sehr gute Korrelation $\left(r^{2}=0.967\right.$, Abb. 3).

Bei 8 Schätzungen wurde die Anzahl Bienen unabhängig von drei Schätzern erhoben. Auch ihre Ergebnisse korrelieren sehr gut untereinander. Das Bestimmtheitsmass $r^{2}$ für die Schätzer $\mathrm{A} / \mathrm{B}, \mathrm{B} / \mathrm{C}$ und $\mathrm{A} / \mathrm{C}$ war $0.991,0.992$, resp. 0.975. Diese Resultate zeigen, dass die Ergebnisse nicht an Aussagekraft verlieren, wenn in einem Versuch verschiedene, geübte Schätzer die Erhebungen durchführen.

\subsection{Brut}

Die Brut wurde sowohl über- als auch unterschätzt (Abb. 4 und 5). Bei der gedeckelten Brut war die Schätzgenauigkeit dank den gut sichtbaren 


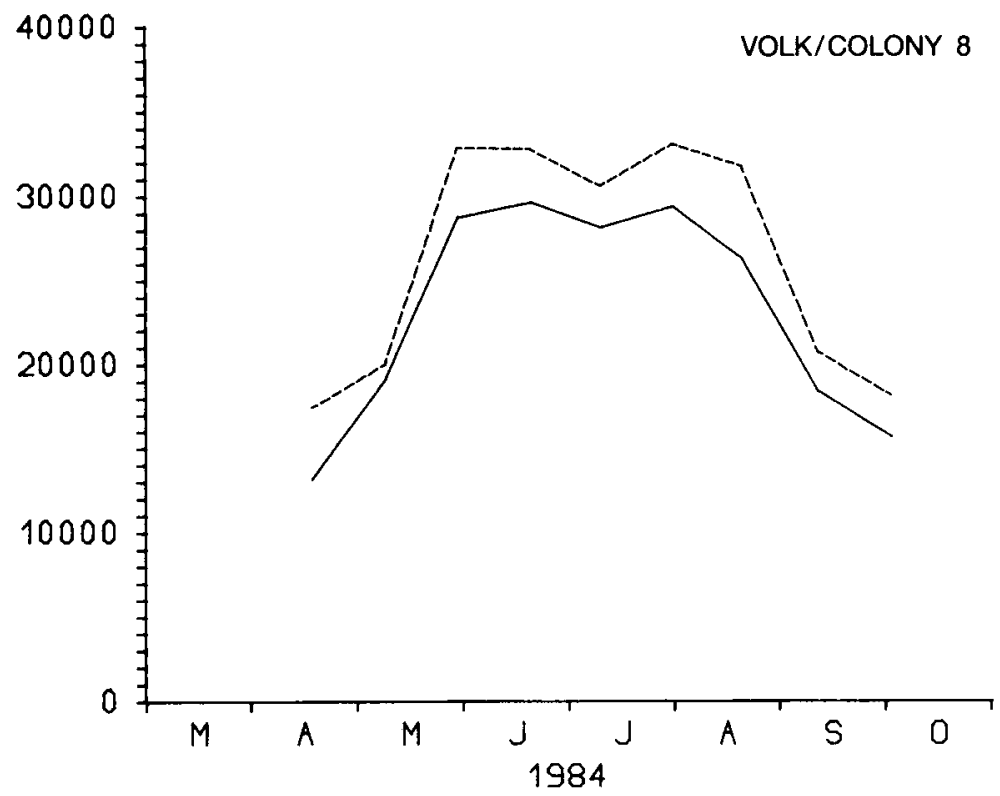

Авв. 2. - Geschätzte (—) und durch Wägen bestimmte (-...--) Anzahl Bienen

FIG. 2. - Number of bees obtained by estimation (_-__) and weighing (-.....--)

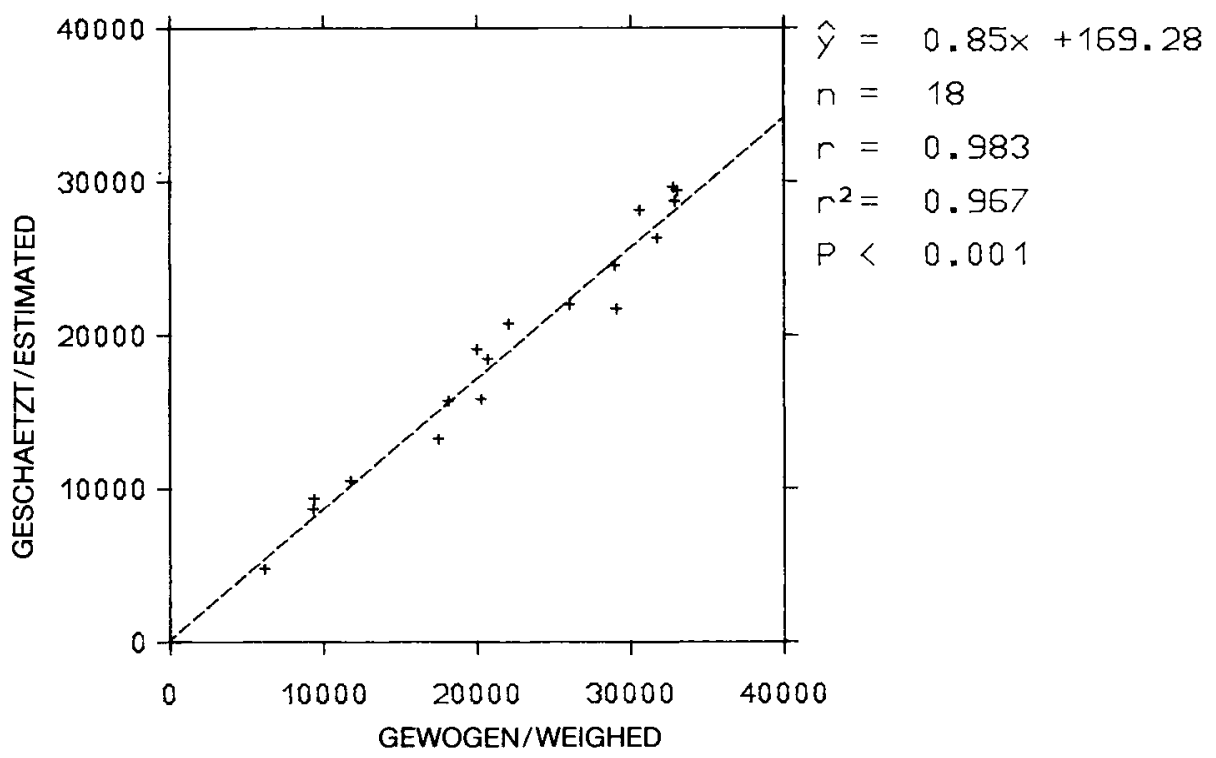

Aвв. 3. - Korrelation zwischen der geschätzten und der durch Wägen bestimmten Anzahl Bienen Fig. 3. - Correlation between estimated and weighed number of bees 


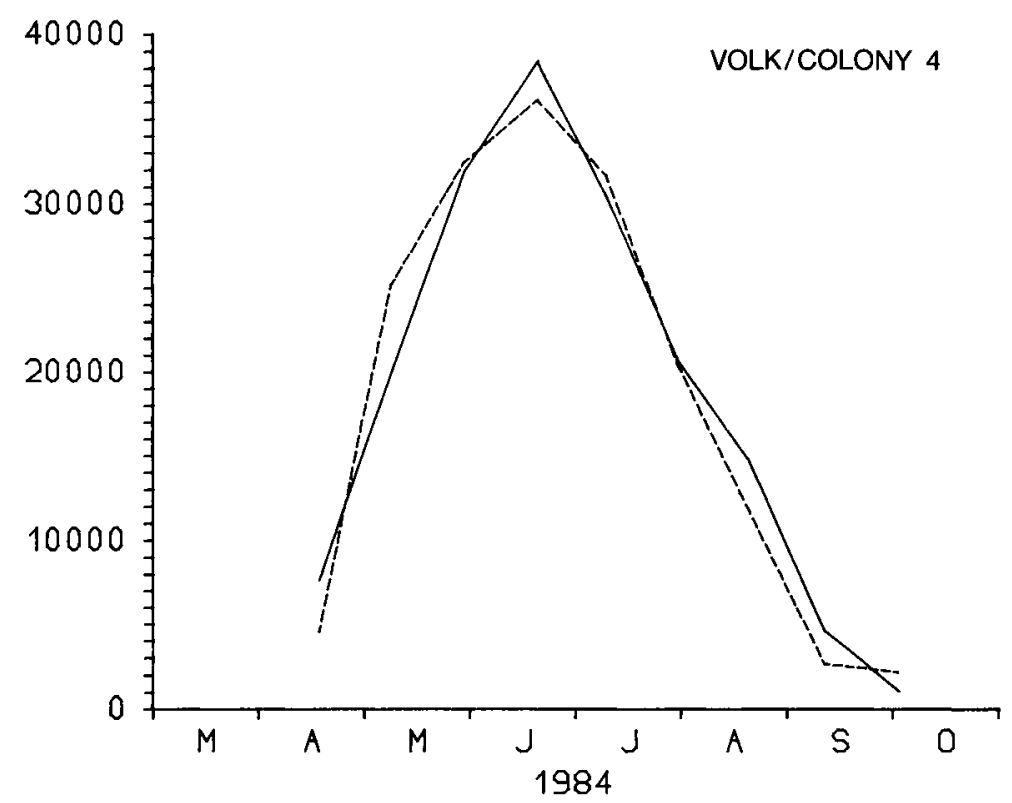

Aвв. 4. - Geschätzte (-) und gemessene (-...-.-.) Anzahl Brutzellen

Fig. 4. - Number of brood cells obtained by estimation (_- and measurement (-........)

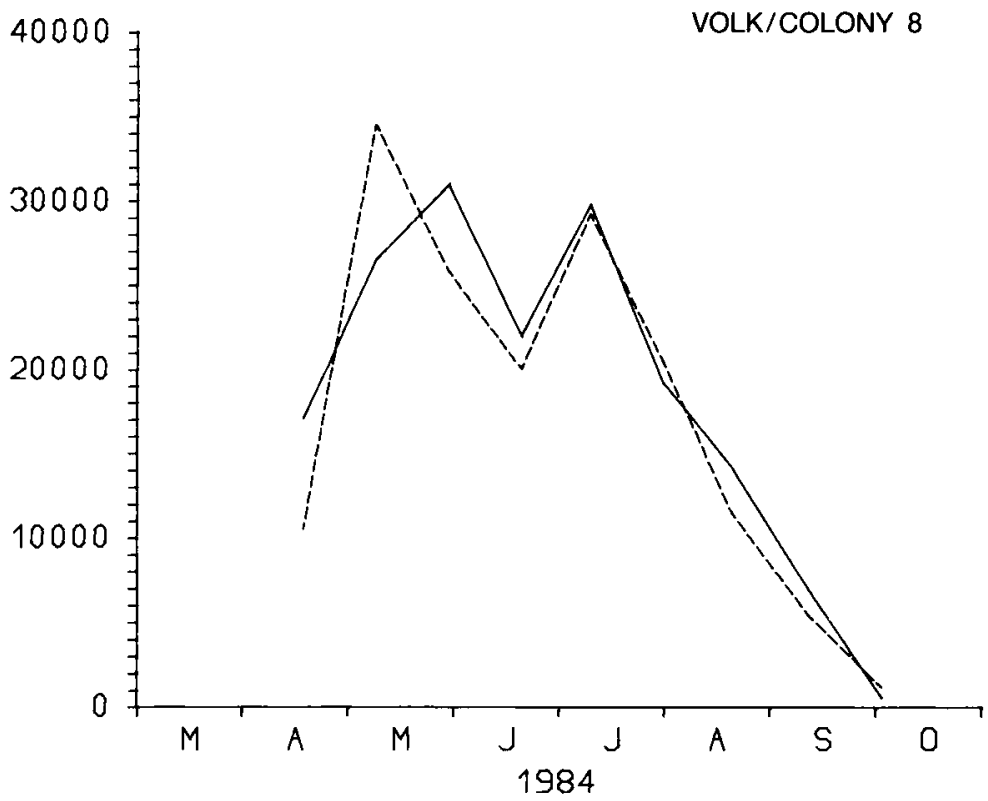

Aв8. 5. - Geschätzte (-_.) und gemessene (-......) Anzahl Brutzellen

FIG. 5. - Number of brood cells obtained by estimation (__ ) and measurement (-......-) 


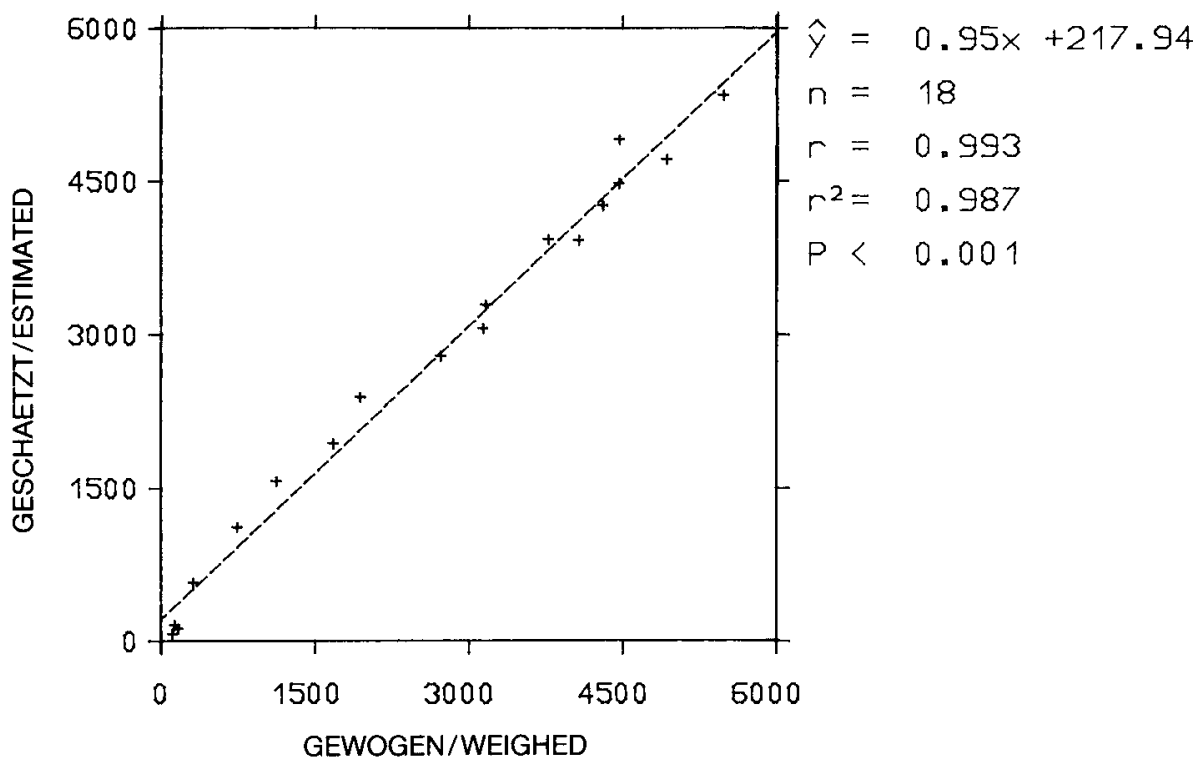

Авв. 6. - Korrelation zwischen den geschätzten und gemessenen, gedeckelten Brutflächen $\left(\mathrm{cm}^{2}\right)$ FIG. 6. - Correlation between estimated and measured areas of sealed brood $\mathrm{cells}\left(\mathrm{cm}^{2}\right)$

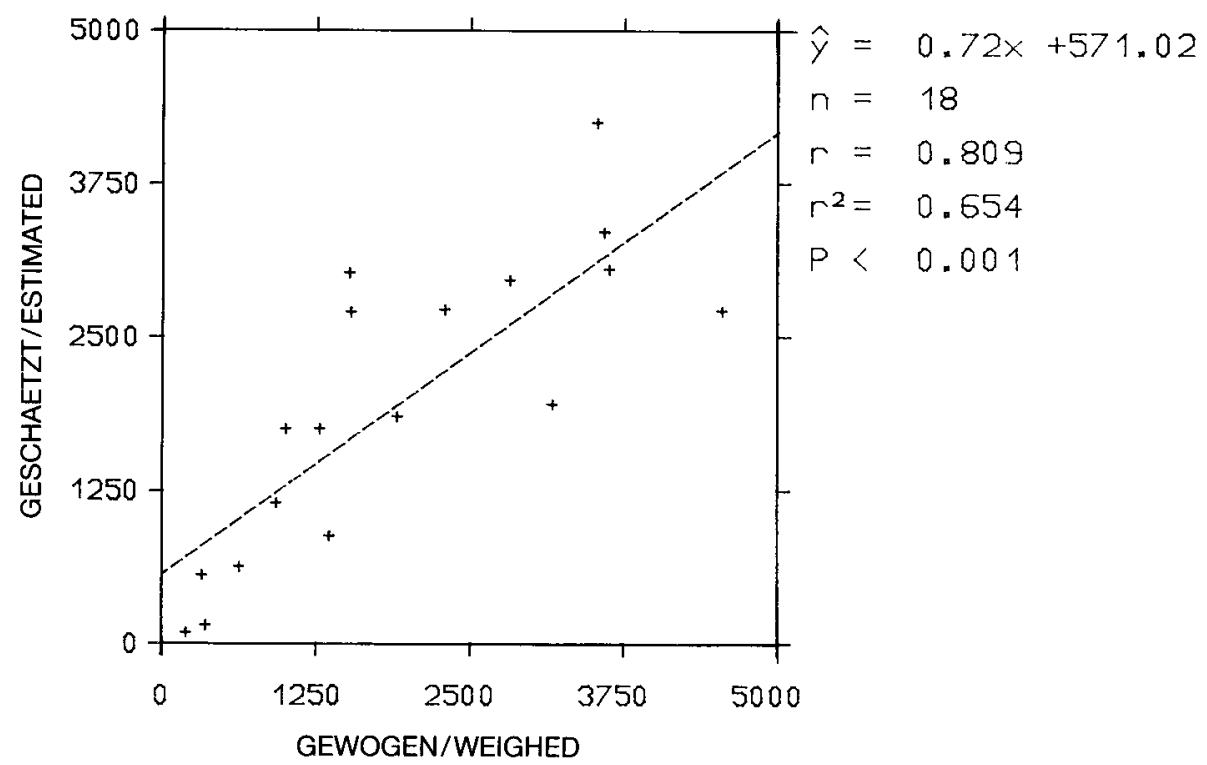

Авв. 7. - Korrelation zwischen den geschätzten und gemessenen, offenen Brutflächen $\left(\mathrm{cm}^{2}\right)$

FIG. 7. - Correlation between estimated and measured areas of open brood cells $\left(\mathrm{cm}^{2}\right)$ 
Flächen sehr hoch $\left(r^{2}=0.987\right.$, Abb. 6). Bei der offenen Brut wurden grössere Schätzfehler gemacht $\left(r^{2}=0.654\right.$, Abb. 7). Das visuelle Erfassen der offenen Brutfläche ist wegen den auf den Waben sitzenden Bienen und den oft ungenügenden Lichtverhältnissen im Bienenhaus schwierig. Vor allem die Flächen mit Eiern sind nicht immer leicht zu erfassen.

Die gesamte Brutproduktion während der ganzen Versuchsdauer wurde bei den Völkern 4 und 8 mit $1.6 \%$ resp. $5.4 \%$ überschätzt. Diese geringen Schätzfehler bestätigen die Brauchbarkeit der Schätzmethode.

\subsection{Bemerkungen zur Schätzmethode}

Wie diese Ueberprüfung zeigt, ist die «Liebefelder Schätzmethode » sehr gut geeignet zur Gewinnung von Rohdaten zum Studium des Massenwechsels freifliegender Bienenvölker. Werden die Schätzintervalle von 21 Tagen eingehalten, so lassen sich anhand von Berechnungen und EDV-Programmen weitere Parameter, wie z.B. das Verhältnis zwischen Brut und adulten Bienen, Gesamtbri tproduktion, das mittlere Alter, die mittlere Lebenserwartung, die Bienentage der Brutrhythmus und vieles mehr bestimmen (BuenlmanN, 1984, 1985, 1986). Der Versuchsansteller verfügt damit über ein Instrument, das ihm besseren Einblick in das dynamische Geschehen des Bienenvolkes ermöglicht. Die Auswirkungen von Pflegemassnahmen, Heilmitteln, Umwelteinflüssen und die Beziehung zwischen Volk und Krankheitserregern lassen sich damit quantifizieren.

Die herkömmliche Methode des Erfassens der Volksstärke mit der Anzahl «bienenbesetzter Waben " oder "besetzter Wabengassen " ist ungenau und führt nach unserer Erfahrung zu beachtlichen Fehlurteilen. Zu völlig falschen Ergebnissen führt es, wenn man sich begnügt, die Volksstärke allein gestützt auf die vorhandene Brutfläche einzuschätzen. Wie anderweitige Auswertungen ergeben haben, besteht nur eine lockere Korrelation zwischen Brutproduktion und Volksstärke (BuehlmanN, 1986).

Der Arbeitsaufwand pro Volk und Schätzung variiert stark nach Jahreszeit, bzw. Volksstärke und Beutesystem. Er beträgt für einen geübten Schätzer zwischen 5 und 15 Minuten. Der Protokollführer kann durch ein handliches und betriebssicheres Tonbandgerät ersetzt werden. Beim Erlernen des Schätzens sind Kontrollmessungen zu empfehlen.

Bei Bienenversuchen werden die Messungen auch tagsüber, d.h. während des Bienenflugs, durchgeführt. Da es aber tageszeitliche, witterungs- und trachtbedingte Veränderungen der Flugintensität gibt, empfiehlt es sich, die Völker verschiedener Versuchsgruppen nicht nacheinander, sondern alternierend zu schätzen. 
Störungen der Volksentwicklung, verursacht durch das vermehrte Oeffnen der Beuten, konnten keine beobachtet werden.

Eingegangen im Juli 1986.

Angenommen im November 1986.

\author{
RÉSUMÉ \\ CONTRÓlE DE LA MÉTHODE D'ESTIMATION DES SURFACES DE COUVAIN \\ ET DU NOMBRE D'OUVRIËRES DANS DES COLONIES VOLANT LIBREMENT
}

La « méthode Liebefeld », développée pour estimer le nombre d'ouvrières et la surface occupée par le couvain, ouvert et operculé, a été réexaminée en 1984. Les contrôles nécessaires ont été effectués toutes les 3 semaines, d'avril à octobre, dans deux colonies volant librement. Nous avons d'abord estimé le nombre d'abeilles et la surface occupée par le couvain, puis comparé ces résultats avec les valeurs obtenues par pesage ou par planimétrie. La corrélation entre les résultats estimés et les valeurs mesurées pour le nombre d'abeilles $\left(r^{2}=0,967 ; n=18\right)$ et pour le couvain operculé $\left(r^{2}=0,987\right)$ est excellente. Pour le couvain non operculé, cette corrélation est plus basse $\left(r^{2}=0,654\right)$. Le total des surfaces estimées pendant l'expérience a dépassé de 1,6\% les valeurs mesurées dans l'une des colonies et de 5,4\% dans l'autre. Les estimations du nombre d'abeilles ont été régulièrement trop basses. Cette divergence peut être corrigée en adoptant comme population standard d'une face de rayon (système suisse $921 \mathrm{~cm}^{2}$ ) 1200 abeilles au lieu de 1 100. Avec cette méthode, il est donc possible de déterminer la dynamique des colonies d'une manière exacte, rapide et sans trop perturber les abeilles.

\title{
SUMMARY
}

\section{A TEST OF THE METHOD OF ESTIMATION OF BROOD AREAS AND NUMBER OF WORKER BEES IN FREE-FLYING COLONIES}

The Liebefeldmethod for estimating the number of worker bees and the areas of open and sealed brood cells was tested in 1984. The estimation were made every three weeks from April to October in two free-flying bee colonies. First we estimated the number of bees and brood areas and then we compared the estimates with the results of weighing or measuring (planimetry). There was a high correlation between the estimated values and the results of measurement for the number of bees $\left(r^{2}=0.967 ; n=18\right)$ and for the sealed brood cells $\left(r^{2}=0.987\right)$. For the open brood cells this correlation was lower $\left(r^{2}=0.654\right)$. The total of these brood area estimates during the test period was $1.6 \%$ higher than the measurements for one colony and $5.4 \%$ higher for the other. The estimate of the number of bees was regularly too low. We were able to correct this by using 1200 bees instead of 1100 for the standard bee population of one side of a comb (Swiss comb $921 \mathrm{~cm}^{2}$ ). This method allows determination of the dynamics of bee colonies accurately, quickly and without major disturbance of the bees.

\section{LITERATUR}

Bruennich K., 1922. - Graphische Darstellung der Legetätigkeit einer Bienenkönigin. Arch. Bienenkd., IV, (4), 137-147.

Buehlmann G., 1984. - Calculating the life expectancy of worker bees (Apis mellifera) by means of the emerging rate and the number of bees. XVII Int. Congr. Entomol. Hamburg, 1984. 
Buehlmann G., 1985. - Assessing population dynamics of a honeybee colony. Mitt. dtsch. Ges. allg. angew. Entomol., (4), 312-316.

Buehlmann G., 1986. - Bienentage - Ausdruck des Leistungspotentials des Bienenvolkes. Schweiz. Bienen-Zeitung, 109, (2), 50-62.

Burgett M., Burikam I., 1985. - Number of adult honey bees occupying a comb : A standard for cstimating colony populations. J. Econ. Entomol., 78, (5), 1154-1156.

Ebert G.R., 1922. - Zur Massenentwicklung der Bienenvölker. Arch. Bienenkd., IV, (1), 1-26.

Farrar C.L., 1937. - The influence of colony populations on honey production. J. Agric. Res., 54, (12), 945-953.

Gerig L., 1983. — Lehrgang zur Erfassung der Volksstärke. Schweiz. Bienen-Zeitung, 106, (4), 199-204.

JEFFREE E.P., 1951. - A photographic presentation of estimated numbers of honeybees on combs in $14 \times 81 / 2$ inch frames. Bee World, 32, (12), 89-91.

JefFree E.P., 1955. - Observations on decline and growth of honcy bee colonies. J. Econ. Entomol., 48, (6), 723-726.

JEFFREE E.P., 1958. - A shaped wire grid for estimating quantities of brood and pollen in combs. Bee World, 39, (5), 114-118.

Nolan W.J., 1925. - The brood-rearing cycle of the honeybee. Bull. u.s. Dep. Agric., $\mathbf{n}^{\circ} 1349$.

Wille H., Gerig L., 1976. - Massenwechsel des Bienenvolkes. IV. Zusammenspiel der Eilegetätigkeit der Königin, der Bienenschlüpfrate und der Lebensdauer der Arbeiterinnen. Schweiz. BienenZeitung, 99, (1), 16-25, (3), 125-140, (5), 245-257. 